\title{
Sexual preference in female rats: II. Choices in tests without copulation '
}

\author{
0. THOMAS LAW, CLAREMONT GRADUATE SCHOOL \\ LAUREN K. GERBRANDT, STANFORD UNIVERSITY MEDICAL SCHOOL
}

A total of 10 female rats was tested for their tendencies to repeat choices in many tests where copulation did not occur. The results indicated that females would avoid repetitive choices to any male if copulation did not occur. Injection of hormones and the presence of other rats in the apparatus did not break up these nonrandom exploratory patterns. After copulation the female rats chose males either at random or repetitively. When males were rotated to corners diagonal to their usual locations the Sprague-Dawley females showed less repetition of choice of the same male, thus further indicating a sexual preference.

"Sexual preference" was used in Experiment 1 to describe the repetitive choices of males by receptive female rats. If repetitions of choice occur when the females are not receptive, or when copulation does not occur, then the preferences are not merely sexual. Beach \& Le Bouef (1967) have found that female dogs tend to prefer the same male dogs only when the females are in estrous. The sexual motivation of rats has, however, been suspected as being basically social (independent of copulation) since rats will barpress to receive other rats regardless of estrous condition or sex of the animal offered as "reward" (Bolles, Rapp, \& White, 1967). Tests of these hypotheses are made in this paper.

Method

The male and female rats used in this study, as well as the apparatus and procedures, are the same as those used in Experiment 1. Four Long-Evans (LE) males, or four LE females, were leashed to the corners of the apparatus. Five Sprague-Dawley (SD) and five LE females served as Ss for preference testing. The main variables studied in this experiment are receptivity of the females and the choice stimuli available in the apparatus corners. The female receptivity and choice stimuli conditions studied were Nonreceptive females-Males Absent from corners (N-MA), Nonreceptive females-Males Present in corners (NMP), Receptive females-Males Absent from corners (R-MA), Receptive females-Males Present in Diagonal corners (R-MPD), and Receptive females-Females Present in corners (R-FP). The N-MA condition was run 4-6 days after testing receptive females in the presence of males in given corners (cf., Experiment 1). One day later the N-MP condition was run. Both of these conditions involved only a single $10 \mathrm{~min}$ trial. These tests were repeated once a week for three weeks. Receptivity or non-receptivity was de- fined by the presence or absence of a lordosis of the female either during or immediately after the 10 min test. The R-MA, R-MPD, and R-FP conditions were run at subsequent one week intervals. The R-MA and R-FP conditions each involved a single $10 \mathrm{~min}$ trial while the R-MPD condition was tested on three trials (10 min each) spaced at 15$45 \mathrm{~min}$ ).

\section{Results}

"Preference scores" of $0,1,2$, or 3 were assigned to each choice as in Experiment 1. The proportions of occurrence of each score are shown in Figs. $1 \mathrm{~A}$ and $1 \mathrm{~B}$ for the various conditions of this experiment. With the exception of the R-MPD condition a typical pattern of choosing among the four alternatives is evident. Females are most likely to visit all other corners before returning to any given corner even though this order of choices is least likely to occur
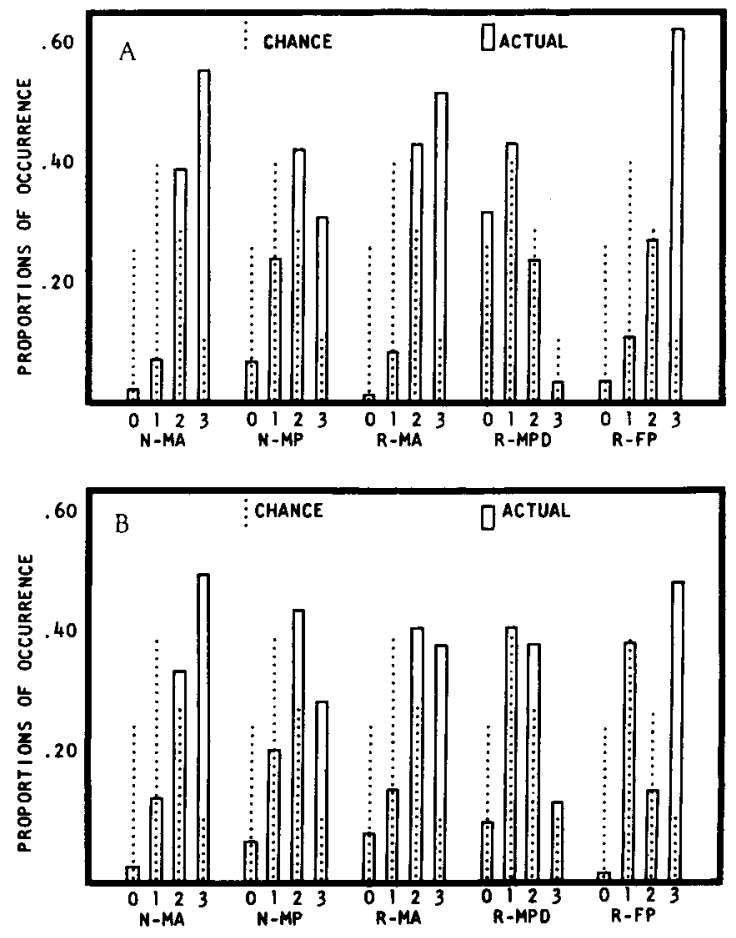

Fig. 1A, B. The proportions of occurrence that $0,1,2$, or 3 other males were chosen before repetition of any choice are shown for the Long-Evans (A) and Sprague-Dawley (B) females in different conditions. N Nonreceptive female, $R$ Receptive Iemale; MA Males absent from comers, MP Males present in comers, MPD Males present in diagonal comers, FP Females present in comers. 
Table 1. $\mathrm{x}^{2}$ and Binomial Probability Results for each Condition

$$
\text { Conditions*** }
$$

N-MA N-MP R-MA R-MPD R-FP Long-Evans Females

\begin{tabular}{|c|c|c|c|c|c|}
\hline Total $x^{2}$ & $355 * *$ & $123 * *$ & $55.3^{* *}$ & $6.1^{*}$ & $100 * *$ \\
\hline Score with greatest $X^{2}$ & 3 & 3 & 3 & 3 & 3 \\
\hline \multirow{5}{*}{$\begin{array}{l}\text { Scores with greatest } \\
\text { probability } \\
\text { Frequency } \\
\text { Binomial probability }\end{array}$} & & & & & \\
\hline & 3 & 3 & 2,3 & 0,1 & 3 \\
\hline & $15 / 15$ & $6 / 14$ & $5 / 5$ & $7 / 11$ & $3 / 4$ \\
\hline & $2 \times 10^{-16}$ & $7 \times 10^{-6}$ & .007 & .24 & .003 \\
\hline & \multicolumn{5}{|c|}{ Sprague-Dawley Females } \\
\hline Total $x^{2}$ & $254 * *$ & $96.9 * *$ & $35.2^{* *}$ & $24.2^{* *}$ & $39.8 * *$ \\
\hline Score with greatest $x^{2}$ & 3 & 3 & 3 & 0 & 3 \\
\hline $\begin{array}{l}\text { Scores with greatest } \\
\text { probability }\end{array}$ & 3 & 2,3 & 2,3 & 0.1 & 3 \\
\hline Frequency & $10 / 15$ & $11 / 14$ & $4 / 4$ & $10 / 11$ & $4 / 5$ \\
\hline Binomial probability & $6 \times 10^{-8}$ & .016 & .019 & .039 & $3 \times 10^{-4}$ \\
\hline
\end{tabular}

* $p .05 ;^{* *} p .001 ; * *$ See Figures $1 A, B$ for Conditions Legend.

by chance. Receptivity, presence of another animal leashed in the apparatus, or corners without males are evidently not sufficient independently to produce the repetitions of choice observed in Experiment 1.

The $\mathrm{x}^{2}$ results for each condition are listed in Table 1. The minimum preference score 3 yielded a higher $x^{2}$ than any other component, except in the case of the R-MPD condition. It should be noted that the SD females, according to the $\mathrm{X}^{2}$ analysis, tend not to return to the same male in the R-MPD condition. The LE females choose males at chance levels during the R-MPD condition.

Essentially the same conclusions are reached using an exact probability binomial model of $\mathbf{n}$ choices for the first four choices of each $10 \mathrm{~min}$ trial. The striking extent of the minimum preference score 3 , or of 2 and 3 , can be seen in Table 1. Only in the R-MPD condition was it necessary to assess the likelihood of occurrence of preference scores 0 and 1. As in the R-MP data reported in Experiment 1, on the first four choices preference occurs at about chance levels for the LE females. As in the previous experiment also, the SD females displayed significant preference during the first four choices of the R-MPD condition.

\section{Discussion}

The results clearly show that the pattern of choices observed in Experiment 1 was appropriately described as "sexual." If copulation occurred during testing, a unique pattern of choosing among alternative corners was seen. In the case of the LE females, males were chosen at random during copulatory tests. It can be seen, however, that in tests where copulation did not occur the LE females were most likely to visit all other corners before returning to any given corner in the apparatus. Receptivity, or the presence of another animal in the apparatus was sufficient to break up this "alternation" or "exploratory" behavior (Dember \& Earl, 1957). These differences in copulatory and noncopulatory tests could be detected even in the first four choices of each test. The SD females showed an even more striking difference in pattern of choices in copulatory and noncopulatory tests. In noncopulatory tests they also were most likely to visit all other corners before returning to any given corner of the apparatus. In later weeks of copulatory testing they did not choose males randomly but were likely to return to the same male. These findings indicate that, in addition to being sexual, the patterns of choice for the SD females are best described by the term "preference." Only when males were rotated to corners diagonal to their usual location were the receptive SD females disturbed in their ability to return to the same males. These findings would be expected if females preferred certain males over others in copulatory tests. Sexual preferences have not been reported previously for the female rat.

\section{References}

Beach, F. A., \& Le Bouef, B. Mating preferences in female dogs. Anim. Behav., in press.

Bolles, R. C., Rapp, H. M., \& White, G. C. The failure of sexual activity to reinforce the female rat. Anim. Behav, in press.

Dember, W. N., \& Earl, R. W. Analysis of exploratory manipulatory and curiosity behaviors. Psychol. Rev., 1957, 64, 91-96.

\section{Note}

1. This research was supported in part by the Hancock Foundation. 